\title{
TECNO-BÍOS: Una aproximación biopolítica a la relación cuerpo-máquina en el contexto cibercultural contemporáneo
}

\author{
TECHNO-BIOS: A Biopolitical Approach to the \\ Body-Machine Relationship in \\ Contemporary Cyberculture
}

\author{
Sebastián Gómez Urra \\ Pontificia Universidad Católica de Chile, Chile \\ seb.gomez.u@gmail.com
}

- Resumen - Las nuevas tecnologías de la información y comunicación forman hoy parte de nuestro entorno, de nuestra naturaleza. En este sentido, vale la pena preguntarse por la relación estética que nuestros cuerpos realizan con los dispositivos, las máquinas, que permiten el acceso a la información digitalizada. El presente artículo responde a los resultados de una investigación bibliográfica en torno a textos sobre biopolítica contemporánea, junto a un análisis histórico de la relación cuerpo-máquina. Los resultados entregan una aproximación a la definición de un tecnocuerpo que se presenta maquinizado, pero que aun así persiste en mantener su carácter de lugar único de producción vital.

Palabras clave: nuevos medios, TIC, biopolítica, relación cuerpo-máquina, cibercultura.

Abstract - Nowadays, the new communication and information technologies are part of our environment, our own nature. Accordingly, it is of worth to wonder about the aesthetic relationship between our bodies and the devices, machines that allow us to access digital information. This article is based on the results given by a bibliographic research about contemporary biopolitics, plus an historical review about the relationship between the body and the machine. Those results deliver an estimate definition of a machinised technobody, although that body still presents itself as the only possible place for vital production.

Keywords: New media, ITC, biopolitics, body-machine relationship, cyberculture. 
Un reciente estudio reporteado por Radio Bío-Bío plantea que la mayoría del tráfico en Internet es no-humano (Ulloa); 51\% de las conexiones realizadas en la red provienen de bots, es decir, softwares que automáticamente recolectan y transmiten información a través de portales de opinión, foros, secciones de comentarios, índices y otros tantos. El estudio establece que un $20 \%$ del total de tráfico en Internet proviene de bots calificados como beneficiosos, pues indexan páginas web en los motores de búsqueda dinámicos que utilizamos hoy -los que, a grandes rasgos, están basados en un ranking en que el primer resultado de búsqueda es aquella página que recibe la mayor cantidad de enlaces desde otras páginas-. Un 31\% son bots maliciosos, espías que recolectan información, "comentadores» que se hacen pasar por personas y difunden spam en los sitios donde se puedan dejar mensajes, junto a un sinnúmero de otras actividades que se resumen, principalmente, en estrategias de mercadotecnia. «El marketing es ahora el instrumento del control social, y forma la raza impúdica de nuestros amos» (Deleuze 3). Un instrumento de control que hoy puede ser delegado a un software que actúa por cuenta propia una vez programado y activado. En este sentido, vale la pena preguntarse por lo benigno de los bots que indexan contenido, cuando, por ejemplo, se sabe que Google recolecta información no solo para mejorar sus motores de búsqueda, sino también para generar la base de datos para marketing más precisa que se ha conocido (Morales). Ese $20 \%$ que se consideraba beneficioso hoy parece sumarse al otro $31 \%$ de bots para generar una mayoría de tráfico no-humano en Internet, dedicado a recolectar información sobre lo que los usuarios humanos hacen en la red. En síntesis, están ahí para el control.

Sociedad de control es un término usado por Foucault para designar un nuevo periodo que se empieza a gestar a principios del siglo $\mathrm{xx}$, cuando el capitalismo se afianza en sus etapas tardías. Se trata de una sociedad en que los medios de producción ya no se concentran en la fábrica, en el encierro -la cárcel, el hospital, la escuela-, como ocurría en la decimonónica sociedad disciplinaria. La inmaterialización del trabajo hace que la administración del poder se efectúe sobre el espacio abierto y se consagre en el control, ya no solo de la fuerza física de trabajo, sino también de la capacidad intelectual de las personas. Es, finalmente, un control absoluto de los cuerpos, en cuanto los medios de producción dominan la fuerza de trabajo en todos sus sentidos, desde la reproducción hasta el pensamiento. Si se retoma el ejemplo del tráfico en Internet, resulta irresistible pensar que es gracias a los nuevos medios -electrónicos, digitales, virtuales, interactivos- que la sociedad de control puede afianzarse. Es a través de las nuevas tecnologías, máquinas virtuales, que se puede controlar la inmaterialidad del trabajo que producen los cuerpos.

El control, de esta forma, parece enviar a la vanguardia a las máquinas que están a su servicio. «La vola terminator las máquinas van a tomar el control de todo [sic]» dice el comentario más votado en la noticia sobre la dominación no-humana del tráfico en Internet. De inmediato aparece en el imaginario el ciberpunk, con películas como The Terminator, como antecedente a lo que podría ocurrir en un 
futuro si es que los grupos económicos que detentan el poder tuvieran la tecnología para dominarlo todo y que, eventualmente, luego las máquinas pudieran derrocar al control humano por su cuenta.

El tópico contemporáneo, el temor que todos parecen compartir, finalmente, es al control. Control que rápidamente pareciera estar nublando los antiguos ciberutopismos que veían en el Internet el lugar para la proliferación de las libertades civiles. Una sensación que se acrecienta cada vez que se escucha en las noticias un nuevo detenido a causa de violar los derechos de autor, como fue el caso de Megaupload, o compartir información privada, como sucedió con Wikileaks.

Un análisis a través de un léxico biopolítico es lo oportuno para lograr comprender esta sociedad de control caracterizada por Foucault. Pero cuando el mundo y, en especial, las tecnologías han cambiado tanto desde la época del filósofo francés, no basta quedarse con su pensamiento para analizar la sociedad contemporánea. Cuando las máquinas se tornan la preocupación central, salta a la vista que el biopoder se canaliza a través de tecnologías, y que es la técnica la que finalmente entregará las herramientas biopolíticas para que la sociedad de control se consagre. Pero, de forma simultánea, un estudio biopolítico sugiere inevitablemente que es al cuerpo hacia donde aquellas tecnologías apuntan, pues es a través de él donde la vida entra en escena. En este sentido, resulta interesante preguntarse por las tecnologías que crean tanto cuerpos como máquinas y que los hacen relacionarse entre sí en el marco de la vida. Se intuye, naturalmente, que es en el ámbito de las comunicaciones, del tráfico de información (como en el caso de la noticia revisada más arriba), que los cuerpos y las máquinas interactúan en una aparente indistinción. En los nuevos medios, máquinas y cuerpos comparten el mismo espacio.

Se tratará de responder la pregunta por los cuerpos, por el cómo son constituidos, moldeados y manipulados, y por cómo ellos también son fuente de creación técnica en su relación con las máquinas con quienes comparten el mundo; por cómo es, estéticamente, este mundo compartido y por cómo es percibido. Para ello, este ensayo deberá alejarse de las teorías que conciben a los nuevos medios como puramente inmateriales, como un texto informativo que debe estudiarse. La relación cuerpo-máquina necesariamente comienza por la materialidad que los conecta, con anterioridad a que ambos se encuentren en la red. Ambos existen como un hardware -orgánico o inorgánico- desde donde producen las inmaterialidades que los constituirán como fuentes y receptores de información.

Finalmente, esta noción de que existe una materialidad perdida llevará a cuestionarse el rumbo que han adquirido los estudios de medios, las ciencias sociales, las humanidades y, en particular, la estética. El estudio contemporáneo de la cultura requiere, necesariamente, de un énfasis en la existencia de ese $51 \%$ con el que compartimos el mundo. Una existencia -su estar-àl-mundo, como diría Nancy- que, además, no puede considerarse sin la materialidad del cuerpo y de las máquinas o, en otras palabras, del cuerpo-máquina. Una relación que implica con-tacto. 
El tecnocuerpo, como figura contemporánea, se torna la imagen central de la sociedad de control. La vida, como lo hubiera propuesto Nietzsche, toma el cuerpo como lugar de batalla entre las fuerzas que la oprimen y mediante las que resiste y avanza. Mas el cuerpo que presenció Nietzsche no es el mismo que se tecnifica en la actualidad. Hoy ese cuerpo dispone de herramientas, maquinaria especializada que le permite constituirse como creación constante, la actualización de su voluntad. El tecnocuerpo podrá aspirar tanto a abrazar su devenir como a volverse obsoleto y perder su capacidad de decir «sí». Es en aquella encrucijada en que resulta relevante encontrar las raíces nietzscheanas del pensamiento biopolítico, pues es finalmente la vida como lucha lo que está en juego.

\section{TÉCHNE DE LOS CUERPOS}

I got the better end of the deal. I only lent you my body you lent me your dream.

Gattaca

Commerce, is our goal here at Tyrell. More human than human is our motto. Rachael is an experiment, nothing more.

Blade Runner

I feel vulnerable... disembodied.

eXistenZ

La relación cuerpo-máquina es tan antigua como el choque entre cultura y naturaleza. El cuerpo, al ser de origen natural pero estar, al mismo tiempo, inserto en la cultura, ha sido un eje problemático fundamental en la tradición occidental. Esta condición física de la existencia heredada de un designio anterior a cualquier intención humana colisiona con los artefactos de los que la humanidad se rodea para dominar a la misma naturaleza que le otorga la vida. La preocupación por la relación cuerpomáquina es inherente al modo en que el ser humano existe en sociedad.

Es en nuestra contemporaneidad cuando aquella relación se vuelve el centro de la problemática. Los antecedentes biopolíticos diagnostican junto al inicio del posfordismo una sociedad en que el trabajo es primordialmente inmaterial, cuando el grueso de los medios de producción se delega a la industria de la comunicación. Las máquinas, paralelamente a los cuerpos, se tornan canales comunicativos, los que en su base necesitan de la conexión expedita entre interfaces humanas y tecnológicas para sustentar el permanente flujo de información entre las diferentes terminales. Los puntos de conexión se multiplican indefinidamente y los modos en que esos enlaces se efectúan se hacen más y más complejos ¿Dónde quedan los cuerpos en esta red de información cuando todo puede entenderse como un flujo comunicativo? ¿Cómo diferenciar, ahora, un cuerpo de una máquina? 
Es en este momento cuando vale preguntarse por la dimensión estética que aparece en la constitución de un cuerpo en permanente conexión con máquinas que le sirven de canal hacia un mundo digitalizado. Los cuerpos, en este caudal de canales informativos, son construidos por la técnica del mismo modo en que se crean las máquinas, pero tal afirmación no parece constituir su homogeneización: sigue existiendo la carne y sigue lo inorgánico sin inmiscuirse completamente en lo orgánico. Tampoco se puede aseverar un distanciamiento entre ellos por el simple hecho de que las técnicas que posibilitan esta manufactura pertenezcan, discursivamente, a disciplinas diferentes. Ambos, en nuestra época, son construidos en base a la posibilidad de su relación, a pesar de que unos sean pensados en la industria cultural y otros en la ingeniería. Ya no parece ser factible hablar de cuerpos sin hablar de máquinas, ni hablar de máquinas sin hablar de cómo ellas se insertan en la sociedad. Pero, de todas formas, es el cuerpo, según el léxico biopolítico, el lugar adonde se dirigen y de donde provienen todas las tecnologías de producción y mantención de la vida. Es en él en lo que debe centrarse un análisis de la sociedad contemporánea.

¿Cómo es, entonces, la vida para este cuerpo que no se entiende sin su conexión a la máquina informativa? ¿Dónde queda la carnalidad del cuerpo en esta aparente anestesia de los artefactos tecnológicos? Podría pensarse que la conexión requiere de la supresión de toda aquella materialidad corpórea que no pueda ser digitalizada y transferida como dato. Pero, tal vez, la codificación del cuerpo responde a otros mecanismos menos evidentes que guardan dentro de sí la posibilidad de un tacto cibernético o una secreción informática. Deconstruir el tecnocuerpo, el cyborg, el hombre postorgánico bajo una mirada biopolítica permite despejar ciertos prejuicios y falsedades que entorpecen la posibilidad de comprender a un cuerpo que recién aparece en la sociedad contemporánea, en la digitalización de la cultura, la constitución de una cibercultura. Un cuerpo que, a pesar de todo lo que pudiera pensarse, todavía acarrea sangre por sus venas.

\section{HISTERIA ANTITECNOLÓGICA}

Muchas de las aproximaciones que han surgido en las últimas décadas respecto al estudio del cuerpo en la era digital responden a un imaginario ciberpunk que concibe el sombrío futuro de la humanidad como uno en que las máquinas tomarán el poder. Estos estudios responderían a la afirmación de que el cuerpo aún sangra con un « ¿y por cuánto tiempo más?». El ciberpunk es la desesperanza de un futuro cercano en que los límites entre humano y máquina se transgreden en formas que inexorablemente llevarán al caos. «El Cyberpunk se trata de expresar ideas (usualmente oscuras) sobre la naturaleza humana, la tecnología, y su respectiva combinación en 
un futuro cercano» ${ }^{1}$ (SFAM, la traducción es nuestra). Pero, más allá del pesimismo que caracteriza a este género de ciencia ficción, está la importancia que tiene su análisis para establecer un léxico capaz de analizar estéticamente las relaciones que existen entre la naturaleza humana y la tecnología, algo en lo que continuamente han fallado las disciplinas más académicas. «El Cyberpunk [...] puede leerse como una nueva forma de teoría social, la cual organiza las consecuencias del rápido desarrollo de la información y la sociedad mediática en la era del tecno-capitalismo» ${ }^{2}$ (Kellner cit. en Lister et al. 318, la traducción es nuestra). Parece relevante, entonces, describir el mundo según el ciberpunk y entender cuánto de ello es aplicable a un análisis contemporáneo de la relación cuerpo-máquina.

Blade Runner de Ridley Scott es una de las películas emblemáticas al momento de establecer una estética del ciberpunk. En ella se descubre la noche, la lluvia, el sol que ha abandonado a la Tierra. Se muestra una iluminación, claramente heredada de los trabajos más lúgubres del cine negro, que hace añorar permanentemente algo de luz natural ¿Dónde está el cielo? La cámara, reticente, se niega a mostrarlo. Tal vez está demasiado contaminado, tal vez sea una visión demasiado triste. Únicamente se limita a mostrar un firmamento negro y sin estrellas, anuladas por la iluminación hecha por el hombre. Siempre es de noche o, peor aún, atardecer. El sol siempre está a punto de irse, a un paso de rendirse para no volver jamás. La luz entra en el cuadro directamente hacia la cámara por pequeñas ventanas y el lente debe forzosamente evitarla para intentar mostrar apenas algo de lo que ocurre. La luz, más que iluminar el espacio, entra como rayos cegadores, alternando frente a los ojos planos completamente blancos o interrumpidos por destellos. "It's too bright in here», se aventura a decir Deckard, para hacer que entre aun menos luz y, paradójicamente, ver mejor. El sol se conservará como algo lejano y extraño. Existirán, entonces, lugares cubiertos que jamás verán el aire libre. No hay naturaleza, pues no sobreviviría en estas condiciones: búhos y serpientes genéticamente construidos serán las mascotas del futuro. Todo está construido con cemento y, sobre todo, con metal. El vapor sale de las alcantarillas colapsadas. Todo está lleno de polvo, sucio, con grasa y desgastado, al menos al nivel del suelo, donde se encuentran los marginados, los protagonistas de estas historias. El único brillo que se encontrará será el que proviene de luces artificiales, tubos de neón y avisos publicitarios. En el peor de los casos, la luz vendrá de componentes radiactivos. Sin embargo, esta falta de luz hará que estas fuentes de iluminación artificiales se vean aún más brillantes. Los edificios, siempre más altos, colaboran a tapar lo que queda de sol. El suelo, húmedo, aloja a lo peor de la población humana, aquella a la que no le fue permitido escapar hacia las nuevas colonias en el espacio exterior. Forzosamente se presenta un espacio antinómico entre fuera y dentro. Un campo de oposición que resulta siempre contradictorio, al igual

1 Cyberpunk is about expressing (often dark) ideas about human nature, technology and their respective combination in the near future.

2 Cyberpunk can (...) be read as a new form of social theory that maps the consequences of a rapidly developing information and media society in the era of techno-capitalism. 
que esa luz que no ilumina nada pero que es tan potente que no deja ver, o como esa cámara que muestra a plenitud la ciudad, pero no a las personas. La mayor parte del tiempo el mundo pareciera estar vacío, las calles solitarias, el edificio abandonado de J. F. Sebastian y, sin embargo, el mercado pareciera estar al borde del hacinamiento. El mundo diegético se muestra así contradictorio y revela realidades opuestas que conviven y se superponen unas a otras, sin suprimirse.

Pero hay algo de sintomático en todo esto. Es sabido que Blade Runner está basada en el libro Do androids dream of electric sheep? (1968), de Phillip K. Dick, y que la novela seminal de la mitología ciberpunk está en Neuromancer (1984) de William Gibson. Ya había, también, una preocupación por la interfaz maquinal en el cine de Fritz Lang y Chaplin a principios del siglo xx . Sin embargo, en los ochenta ocurrió un giro dramático que preocuparía a toda la sociedad que hará que lo relevante sea preguntarse qué es la humanidad. La ingeniería genética y la realidad virtual empiezan a tener una visibilidad vertiginosa. Se ataca a la realidad humana desde dos frentes: desde su creación y desde el ambiente donde habita, ya que ambos pueden ser fruto de la fabricación técnica. Nace, de esta forma, la posibilidad pronta de un hombre pos-orgánico o un pos-humano que deseche las viejas limitaciones de la naturaleza. Asimismo, la mayoría de las producciones artísticas centradas en esta temática sitúan su universo diegético en la inmediatez del mañana: es fundamental emplazar al espectador en una posibilidad cercana de peligro. En general, aquel futuro cercano no se radica más allá de un par de generaciones alejadas de la actual. Siempre habrá alguien conocido, un hijo o un nieto, que vivirá el posible desastre en que los grupos de poder se adueñan de la tecnología que es capaz de crear realidades. La mayoría de las películas, videojuegos y libros inspirados por el ciberpunk llevarán al espectador a encontrarse con un mundo en que una tecnología específica ha causado un desastre. Las consecuencias de la ruptura de la armonía serán devastadoras. La Tierra, como planeta, cambiará de tal forma que la vida humana deberá adaptarse a nuevas circunstancias que antes no conocía. Habrá saqueos, hambruna o una nueva amenaza a la vida: enfermedades, epidemias o una especie alienígena distinta y superior a la humana.

Y así, en los orígenes de este movimiento estará Blade Runner centrando en sus personajes preguntas existenciales anteriores a la posible mezcla: el reemplazo, la réplica. ¿Por qué habrían de seguir existiendo humanos si a través de la técnica se pueden crear otros mejorados? O incluso ¿cómo saber qué es lo humano?, ¿cómo saber si soy humano o no? Somos, finalmente, construcciones replicables y, una vez que es posible entender cómo funciona la memoria, nuestros recuerdos pueden ser creados virtualmente. ¿Dónde queda entonces lo que es humano? ¿En la creación? ¿No serían humanos las concepciones in vitro? ¿En los sentimientos, entonces?: reacciones químicas manipulables.

La campaña épica de Deckerd, el héroe mítico en busca de la restauración del orden original, pierde validez en el momento en que los pares antagónicos se disuelven 
en nuestra contemporaneidad. ¿Cuál es el orden que debe restaurarse? No existe una base sobre la cual establecer líneas divisorias entre humano y no humano, dentro y fuera, soledad y hacinamiento, visibilidad y oscuridad, vida y muerte. Sutilmente, Scott nos hace entender que Deckerd es lo mismo que intenta cazar. ¿Sueñan los androides con ovejas eléctricas? ¿Qué hacer cuando el mismo restaurador mítico es cuestionado en su esencia? No hay un "otro» contra el cual luchar, contra el cual definirse. Los conceptos binarios se superponen multifocalmente. Se ramifican y separan en nuevas reproducciones, se replican. Las contradicciones viven sin oposición.

Lo que se intenta cuestionar aquí es la construcción del propio cuerpo. Ya no hay propiedades únicas del alma; todo es parte del mismo organismo, propio de su factura. ¿Qué importa si se es un cyborg o un androide? La artificiosidad del cuerpo ya no es contraria a su naturalidad. Lo natural ya no es sinónimo de humanidad. Sudor, sangre, semen, amor, odio, ira, locura, nostalgia; los Nexus6 son capaces de todo eso y más.

El ciberpunk no entrega solución más que el éxodo hacia el ciberespacio, la conquista de las tecnologías más allá de las intenciones del tecnocapitalismo. Algo así como lo que ahora hacen agrupaciones como Anonymous, quienes intentan mantener a toda costa la libertad de opinión y de información en Internet. Pero este tipo de luchas olvidan -tal vez justificadamente- el carácter material del mundo que habitan y de las tecnologías que dicen controlar. A pesar de ello, el ciberpunk permite establecer un léxico que marcará los estudios de los nuevos medios hoy: ciberespacio, realidad virtual, cyborg. Si bien no todos estos términos tienen su origen en la producción artística, es ella la que toma el vocabulario científico y lo establece como denominador común a varios ámbitos de una cibercultura. Consecuentemente, el ciberpunk marcará, en sus momentos más sombríos, el tono que la investigación académica tomará hacia la temática de la relación futura entre cuerpo y máquina.

Paula Sibilia, en su libro El hombre postorgánico, escribe:

En la actual «sociedad de la información», la fusión entre el hombre y la técnica parece profundizarse, y por eso mismo se torna más crucial y problemática. [...]. En este contexto surge una posibilidad inusitada: el cuerpo humano, en su anticuada configuración biológica, se estaría volviendo obsoleto. Intimados (y seducidos) por las presiones de un medio ambiente amalgamado con el artificio, los cuerpos contemporáneos no logran esquivar las tiranías (y las delicias) del upgrade. Un nuevo imperativo es interiorizado: el deseo de lograr una total compatibilidad con el tecnocosmos digital (12-3).

Para ella, el cuerpo ya ha perdido la batalla en lo que se refiere a su relación con las máquinas. De partida lo presenta como una lucha, un antagonismo que no se justifica más que por su separación originaria, como si la diferencia entre orgánico e inorgánico fuera suficiente para causar un conflicto en que solo uno puede ser el vencedor. Esta visión, tal vez, tenga mucho que deberle a la apreciación marxista de las máquinas como seres alienantes para el trabajador. Pero comparte, además, una cierta tonalidad preapocalíptica con el ciberpunk. Esta aproximación, de todas formas, no 
es única a Sibilia, como lo estudiarán Lister et al. en New Media. La teoría de medios y las ciencias sociales en general, sobre todo aquellas bajo la influencia de Raymond Williams, tienden a contraponer un determinismo tecnológico con un humanismo en peligro de extinción. En un tono de alerta, casi heideggeriano, remarcan los cuidados que la humanidad debe tener para mantener a la técnica bajo control. No quiere decir que las ciencias sociales prevean un escenario como el de los filmes The Terminator o I, Robot; pero sí basta para ellos nombrar el rumbo que la biotecnología y la ingeniería han tomado: eugenesia, Proyecto Genoma Humano, bombas nucleares, control satelital. Sus reparos no dejan de ser sinceros, mas muchos de ellos pecan de «histeria antitecnológica» como diría Sloterdijk (cit. en Sibilia 195). No obstante, no hay otra salida que una catástrofe en el cruce de materia orgánica e inorgánica cuando ambas se consideran parte de mundos opuestos e irreconciliables. Un análisis biopolítico podría entregar herramientas para considerar que tal oposición nace únicamente con el advenimiento de la vida en el terreno político y la subjetivación del cuerpo mediante dispositivos técnicos. Antes de eso, antes del nacimiento del capitalismo, no existía una zoé atravesada al bíos que pusieran a lo orgánico en ámbitos disciplinarios diferentes al interés de la ingeniería, a pesar de que los medios y objetivos, técnicos todos, fueran esencialmente los mismos. La biotecnología de las últimas décadas no viene más que a reforzar esta aseveración. Es necesario destacar, sin embargo, que la biotecnología o bioingeniería no son en ningún caso una vuelta al pensamiento del engranaje de la primera modernidad que concebía al cuerpo orgánico e inorgánico iguales en estructura. El bíos no estaba presente, en ese entonces, como lo está en esta nueva disciplina. Antes no se intentaba crear una vida mejor, sino que se esperaba simplemente lograr un funcionamiento equivalente.

El pensamiento en la época del engranaje da cabida, empero, al dualismo que salvaguardará la integridad humana ante la técnica y la opondrá desde ese momento en adelante. El cartesianismo, con la separación definitiva entre razón y cuerpo, dejará a este último en las manos de la experimentación técnica y, además, posibilitará comprender al ser humano como un sujeto independiente, no limitado en su ser a las mundaneidades de su constitución material. En las sociedades anteriores al nacimiento de la vida -y, con ello, de la biopolítica- había sido algo común la noción de que el entendimiento del pensamiento, de la razón y de las demás facultades ligadas al alma pertenecían a un ámbito del conocimiento relacionado a la contemplación y el intelecto. Descartes, por su parte, sitúa a la razón en una relación dual con el cuerpo, pero no determinada en absoluto por este último. El ser humano no se concebía sin su res extensa, pero se establecen competencias que son independientes entre ambas partes de la existencia del hombre. Con la entrada de la vida en la escena, la razón peligra en su independencia en cuanto ella empieza a estar determinada por la capacidad del cuerpo de mantenerse con vida. Algo que la ciencia tempranamente se dará cuenta de que no puede hacerse sin la necesaria ayuda -tecnopolítica- externa que pudiera mantener al cuerpo saludable en sus capacidades físicas y mentales. 
El dualismo cuerpo / razón se extremará con el surgimiento del capitalismo, siglos más tarde. Es más, podría decirse que una de las características de la sociedad disciplinaria en el capitalismo temprano será el separar el cuerpo material del trabajador como estadística controlable, alejado de su individualidad intelectual. Alienado a la producción, el cuerpo se vuelve un anexo circunstancial no muy diferente del resto del engranaje, cuando, al mismo tiempo, se le otorga al intelecto las labores que las máquinas-cuerpo no pueden suplir. La creciente importancia de las labores inmateriales para asegurar una mejor empleabilidad lleva a pensar el cuerpo como una limitante. Será esto lo que Sibilia, en base a las distinciones de Hermínio Martins, caracterizará como una visión fáustica de la técnica: «La tecnociencia contemporánea constituye un saber de tipo fáustico, pues anhela superar todas las limitaciones del carácter material del cuerpo humano, a las que entiende como obstáculos orgánicos que restringen las potencialidades y ambiciones de los hombres» (43).

Asimismo, la irrupción biopolítica del capitalismo, mientras más se acercaba a sus dinámicas posfordistas, más hacía pesar la balanza hacia las limitantes corpóreas. La razón, ahora necesariamente ligada a una vida orgánica, necesitaba controlar la materialidad de su existencia. Las esperanzas de que la técnica pudiera superar las barreras del frágil cuerpo lleva a un utopismo frenético en el que se alcanzara una existencia puramente inmaterial. Como dirá Foucault, con la irrupción de la vida en el hombre ya no es el alma la que se considera encerrada en el cuerpo, sino que la relación se invierte: «El alma, efecto o instrumento de una anatomía política; el alma, prisión del cuerpo» (Vigilar y castigar 36). La importancia de la información como inmaterialidad hará que el cuerpo, en su espacialidad y temporalidad, se sienta infinitamente inferior, debiendo adaptarse técnicamente a la capacidad vital del intelecto, no al revés. El cuerpo deberá inmaterializarse, condensarse y moverse rápidamente para alcanzar las posibilidades que a la razón se le han entregado mediante las nuevas tecnologías. «El cuerpo significante -todo el corpus de los cuerpos filosóficos, teológicos, psicoanalíticos y semiológicos- solo encarna una cosa: la absoluta contradicción de no poder ser cuerpo sin serlo de un espíritu que lo desincorpora» (Nancy, Corpus 55).

Las nuevas tecnologías parecerían, bajo esta perspectiva, posibilitar la inmaterialización del cuerpo en pos de una vida absolutamente mental. "A medida que interactúo con la Red, me reconfiguro a mí mismo; mi extensión-red me define exactamente como mi cuerpo material me definía en la vieja cultura biológica; no tengo ni peso ni dimensión en cualquier sentido exacto, solo me mido en función de mi conectividad» dirá el representante del tecno-art Ray Ascott (cit. en Sibilia 51). Los nuevos medios parecerán posibilitar una conexión que se entiende comúnmente como exclusivamente virtual: una réplica del mundo real pero que permite abandonar las limitaciones del cuerpo y transferirse mentalmente al plano cibernético. «Ciberespacio» llamará William Gibson en Neuromancer a este lugar. Esta utopía -el no-lugar que es un mejor lugar-, será lo que el ciberpunk constatará como la 
posibilidad de salvación del caos que el futuro postapocalíptico dejará en la «vida real». Se entiende, entonces, que el castigo mayor en el universo de Neuromancer sea estar condenado a la vida material, sin posibilidad de conexión al ciberespacio.

La utopía del ciberespacio se mantiene virginal en los ochenta, pero decae en el momento en que los hackers se dan cuenta de que ese lugar ya no les es propio, pues las empresas y corporaciones lentamente van dominando el contenido que puede y no puede existir en la red. La sociedad de control entra en acción. «En las sociedades de control [...] lo esencial no es ya una firma ni un número, sino una cifra: la cifra es una contraseña [...]. El lenguaje numérico del control está hecho de cifras, que marcan el acceso a la información, o el rechazo» (Deleuze 2). Matrix, un filme de los hermanos Watchowski, presentará, a principios del siglo xxi, al ciberespacio como un lugar de lucha y resistencia. La vida real ya no es el castigo, es la verdad. Conocerla o no depende de qué color de pastilla se elija. Pero se ha invertido el movimiento de Neuromancer. El estado original ya no es en la vida real, sino en el ciberespacio. Tomar la pastilla roja es el acto consciente de querer conocer la materialidad oculta, perdida. Es el inverso de la caverna platónica. "Where are our real bodies? Are they alright? What if they're hungry? What if there's danger? " ", se pregunta Ted Pikul en eXistenZ luego de pasar demasiado tiempo al interior del ciberespacio en su primera sesión de videojuegos.

El Fausto, que intentaba por todos los medios deshacerse de su cuerpo, parecer tornarse obsoleto cuando se percibe al cuerpo el lugar al que siempre se retorna, el lugar único que permite la vida. Es más, Marshall Berman relee el mito fáustico como una tragedia que permite entender el surgimiento del capitalismo bajo la misma terminología. Fausto debe asumir que su poder diabólico no es una opción, es un deber. Un deber que lo llevará a destruir constantemente para crear el futuro. «Esta es la dialéctica que pronto envolverá y moverá a toda la economía, el Estado y la sociedad modernos como un todo» (Berman 40). Serán el cuerpo y su intelecto, dirá la biopolítica, las armas que irreversiblemente deben explotarse y poner su capital en circulación, en constante creación y destrucción. Este será el «desarrollo» de la sociedad capitalista, pero también su condena. Fausto no avanza con malas intenciones, pero serán estos objetivos los que inevitablemente terminarán por destruirlo todo. Querer siempre más no es una decisión que pueda negarse, es la condición misma de la existencia moderna y lo que lleva a la explotación inagotable del propio cuerpo y la mente. La figura del Fausto, como parece plantearla Sibilia y otros, «aparecía en un nuevo papel simbólico, como el demonio que había privado a la humanidad de su unidad primigenia con la naturaleza y nos había empujado por el camino de la catástrofe» (Berman 76). Pero esta figura -más melodramática que trágica, según Berman-, plantea ingenuamente la posibilidad de un regreso a una sociedad en que existe una potencia vital diferente a las técnicas que la producen:

¿Donde están nuestros verdaderos cuerpos? ¿están bien? ¿qué pasa si tienen hambre? ¿qué pasa si están en peligro? 
«Las tecnologías de producción de almas y cuerpos, en todos los tiempos, suelen conspirar contra las potencias de la vida [...]. Pero la vida opone resistencia a los dispositivos desvitalizantes y siempre es capaz de encontrar nuevas fuerzas» (Sibilia 100). ¿Cómo puede entenderse una sociedad biopolítica junto a dispositivos desvitalizantes? ¿Qué esencialidad puede tener la vida que no sea otra que las propias técnicas de producción?

La tragedia fáustica es esa: una vez se hubo descubierto una tecnología ya no se puede desconocer, pues ella, en su aparición, modifica las técnicas de producción vitales. Lo importante de Fausto para Berman está precisamente en el desarrollo que el mismo personaje va teniendo en la apropiación de las capacidades de su nuevo ser. Es necesario asumir, entonces, que no existe un «desarrollismo» que no lleve en sus reflexiones el conocimiento de un mundo material que está forzando a cambiar. Las decisiones fáusticas comprenden irrenunciablemente transar desde un rol técnico con situaciones reales, otros cuerpos y tecnologías, leyes, Estados y empresas, en que el acuerdo de si vender el alma al diablo o no se toma en cada instancia, en la que modifica su propio ser en cada acción tecnocreativa que realiza. Eso es la vida contemporánea, no la madre naturaleza.

De esta forma, el antagonismo entre cuerpo-natural y máquina-artificial no es sostenible como línea de reflexión en la sociedad moderna. La discusión se vuelve inviable cuando cada nueva tecnología irrumpe, como diría McLuhan, en el ecosistema del ser humano y lo modifica de tal manera que la sociedad debe reaccionar y poner en la balanza lo que está dispuesta a transar para hacerle un lugar en el mundo. La tecnología se hace parte indistinguible del propio cuerpo en cuanto se hace un ente con el que se cohabita y con el que tiene que dialogar de forma constante.

Nuestro mundo es el mundo de la «técnica», el mundo del cual el cosmos, la naturaleza, los dioses, el sistema completo en su juntura íntima, se expone como «técnica»: el mundo de una ecotecnia. La ecotecnia funciona con aparatos técnicos, a los cuales ella nos conecta desde todas partes. Pero lo que ella hace son nuestros cuerpos, a los que pone en el mundo y conecta a este sistema [...]. En la creación de los cuerpos es donde la ecotecnia tiene este sentido que se le busca en vano en los restos de cielo o de espíritu (Nancy, Corpus 69).

Al modo de McLuhan, Jean-Luc Nancy sitúa al cuerpo en un mundo cuya ecología es construida por la técnica. Mas no es el cuerpo lo creado por la técnica, es el cuerpo el que se hace en la creación. El mundo entero es creado técnicamente como mundo de cuerpos que se hacen únicos en su multiplicidad. Bajo esta perspectiva, el alma, la razón, el intelecto, se hacen a la vez cuerpos creados ecoténicamente. «Psyche es extensa»: la cita de Freud que reproduce Nancy. Una aseveración que inevitablemente ya estaría intuida en Descartes, cuando se sigue que el alma se conoce en su extensión según la propia extensión del cuerpo, y no a través de él (Nancy, 58 indicios 41). El alma se vive en la extensión a la que su inextensión está unida como creación. No hay otra posibilidad para ambos de estar en el mundo. Esposito 
criticará a Nancy el haber rechazado la carne por ser el par dialógico del espíritu y, por lo tanto, contenerlo (Esposito 260), pero para Nancy no es posible reducir el cuerpo a la carne: «El cuerpo es una prisión o un dios. No hay término medio. $\mathrm{O}$ bien el medio es un picadillo, una anatomía, una figura desollada, y nada de eso hace cuerpo» (58 indicios 16). La biopolítica no está involucrada con la carne, sino con los cuerpos, con la creación técnica por la que los cuerpos están hechos y a través de la que los cuerpos hacen. En la carne hay una dualidad que el cuerpo no admite. El cuerpo no encarna sentido, sino que crea mediante la secreción: secreta palabras, ideas y sangre, ninguna de ellas menos corpórea que de donde proviene.

Finalmente, no es posible pretender que hay una separación que pueda lograrse, o que la biopolítica pretenda lograr, que sustente mediante la utilización de técnicas inmaterializadoras una destrucción de la materialidad del cuerpo en favor de lo preponderante del alma / intelecto / razón. Nuestra naturaleza es técnica y bajo esa premisa es que un análisis de la relación cuerpo-máquina en la sociedad contemporánea debe hacerse. En consecuencia, la «histeria antitecnológica» no se justifica bajo ningún sentido cuando es nuestro propio cuerpo el que, en su composición, es un fruto de la tecnología. Y el cuerpo, a su vez, no tiene otra forma de moverse por el mundo que creando técnicamente otros cuerpos, sean estos orgánicos o inorgánicos.

Biopolíticamente, se entiende que para la sociedad actual no puede haber una naturaleza que sea distinta a la que es configurada en la vida de un cuerpo -sin importar si ese cuerpo es equivalente a un hombre, un pueblo o un gobierno-. Por lo tanto, cuando se observa la relación cuerpo-máquina, en términos en los que ambos son tecnología, se les considerará del mismo modo a ambos simultáneamente cuerpos, y su relación tendrá la característica de ser una comunicación directa entre miembros de una misma categoría.

\section{VIDEOJUEGOS Y CIRCUITOS CUERPO-MÁQUINA}

Cuando la histeria se apodera de las lecturas de las ciencias sociales y las humanidades hacia las tecnologías, es necesario tornarse hacia otras formas estéticas de relación cuerpo-máquina en la cultura. El ciberpunk, como categoría estética, sin embargo, aún registra posibilidades que pueden ser de utilidad a este tipo de análisis. Al modo en que se presentaba más arriba, el ciberpunk acarrea una terminología que permite comenzar a pensar en la superación de los dualismos occidentales. Este género, a pesar de ello, sumido en la esperada derrota ante el avance -y magnificencia- de las máquinas, sufrió una transformación paulatina hacia otras problemáticas conforme acababa el siglo xx . Por ejemplo, considerar el ciberespacio como lugar distinto al plano material parece, en la actualidad, tornarse cada vez más alejado del futuro humano. La Realidad Virtual (VR, por sus siglas en inglés), antes se imaginaba como un entorno completamente ajeno al cuerpo, en el que se digitalizaba un nuevo cuerpo simulado para la mente independiente. Es una imagen popularizada por el ciberpunk, 
en el que una conexión física -un cable insertado en el cuerpo (Matrix, eXistenZ), o un casco y un guante, en sus versiones más realistas- suprime al cuerpo material y transporta a la mente en forma independiente al ciberespacio. «[H]oy asoma una especie de neocartesianismo high-tech, en el cual la vieja oposición cuerpo-alma correspondería al par hardware-software» (Sibilia 86).

Pero la realidad virtual dejó de ser, en este aspecto, tema central para la ingeniería informática. Se habla ahora de Realidad Aumentada (Augmented Reality) en cuanto ya no se pretende reemplazar una realidad con otra, sino que ambas -mundo real y mundo generado computacionalmente- se complementan y se superponen. Esto podría explicarse como consecuencia de que en la actualidad la tecnología digital se percibe como parte del medio ambiente y se invisibiliza su presencia, en términos mcluhianos. La conexión con los objetos que permiten, a su vez, la conexión a mundos digitalizados se asume de forma permantente, cuando se vuelve accesible en cualquier momento o lugar. Ya no es necesario llegar a hot spots, pues el enlace puede hacerse de manera celular o satelital. Así, insertarse un cable en la espina dorsal para conectarse a un videojuego parece una idea absurda de futuro.

Sin embargo, los videojuegos aún son el mejor ejemplo de cómo se da ese enlace en la actualidad entre cuerpo y máquina. El ciberpunk ve en ellos una posibilidad cierta de la existencia del ciberespacio como lugar alternativo a la realidad. Tron, de Steven Lisberger, por ejemplo, trata de un programador de videojuegos que logra no solo insertar su mente en el mundo virtual, sino el cuerpo en su totalidad, el que termina desapareciendo del mundo factual. De hecho, fueron los primeros hackers los que lograron que el antiguo uso de los computadores como máquinas de oficina se transformara en medio de entretenimiento, al forzar la programación de ellas en lo que serían los primeros videojuegos. La creación de mundos virtuales será, por lo tanto, el primer uso social no previsto por los desarrolladores de la tecnología computacional y será, finalmente, este uso el que dará origen a fantasías como la de Tron, eXistenZ y el dualismo virtual / real.

Una vez se hubo desistido de la noción de realidad virtual como opuesta a realidad sin adjetivo, los videojuegos continuaron siendo un lugar de creación de mundos permanente. Entornos diegéticos que lograrán una sensación de inmersión en el espectador / jugador que el cine nunca pudo siquiera llegar a soñar. Hoy, la historia de un videojuego puede llegar a durar más de cien horas, o continuar indefinidamente, como es el caso de los Massively Multiplayer Online Games (MMOG). La relación cuerpo-máquina adquiere ribetes insospechados, ni siquiera pensados por el ciberpunk. Mas ¿qué es lo que hace que los videojuegos ya no necesiten de la realidad virtual para lograr una inmersión que puede interrumpirse en cualquier minuto pero seguir siempre vigente? ¿Por qué los lentes $3 \mathrm{D}$, el sonido surround, o las vibraciones en el controlador parecen ser accesorios complementarios y no son elementos esenciales para los gamers? 
La noción de phylum maquínico de Deleuze y Guattari podría entregar algunas luces sobre este cuestionamiento: «No estamos usando una metáfora, sin embargo, cuando hablamos de máquinas; los humanos consitutyen una máquina tan pronto su naturaleza sea comunicada por repeteción al conjunto del cual forman parte bajo condiciones específicas ${ }^{4} »$ (Guattari 131, la traducción es nuestra). Sin embargo, esta aseveración no tiene las mismas implicaciones que aquellas propuestas por Marx. Lo que Deleuze y Guattari proponen no es que las máquinas hayan alienado al hombre y lo hayan fagocitado dentro de su funcionamiento. Ellos sugieren que tanto los seres humanos como las máquinas pertenecen al mismo orden taxonómico, un phylum que los clasifica bajo la misma rama, anterior a su división en especies distintas. Lo que caracteriza a ambos es su repetición y su comunicabilidad. Una comunicabilidad recurrente que, según lo desarrollado más extensamente en el Anti-edipo, mantiene un flujo constante entre los distintos elementos de la máquina social. Deleuze, por su parte, en sus libros sobre cine advierte la formación de un circuito entre el espectador y el filme (Lister et al. 384). Pero aquel circuito es el más corto posible formado en el feedback entre estímulos sensoriales y cuerpos orgánicos. Logran modelar así un único sujeto maquínico que no es ni la proyección fílmica ni el espectador, sino el conjunto de circuitos entre ambos. Sin embargo, lo que Deleuze y Guattari proponen, y que los aleja definitivamente, tanto de Marx como de un determinismo tecnológico, es que lo maquínico en cuanto phylum entra a una clasificación biológica más que técnica, o, en otras palabras, la técnica misma es comprendida solo a través de un orden natural. La máquina de deseo, entonces, se explica no como un sujeto que busca satisfacerse en la obtención del objeto, sino como un creador de pequeños circuitos con el mundo que agencia y reordena permanentemente a su alrededor. La máquina es el agente desterritorializante que permite que el mundo se autoorganice de forma constante, sin importar si los elementos que componen el circuito son orgánicos o inorgánicos. Este dinamismo presupone, entonces, que la máquina misma es un circuito en constante reformación y, por tanto, su funcionalidad permanece indeterminada. No hay posibilidad de determinismo tecnológico cuando la propia tecnología permanece sin determinación. Lo mismo ocurre cuando se intenta afirmar un determinismo social: no hay sujeto que pueda desear un único uso para una tecnología, al modo en que la tecnología en su funcionamiento no presupone el circuito que formará con el resto de máquinas -sociales y de deseo- que lo rodean.

La noción de virtualidad, como realidad alterna a la verdadera realidad, ya no puede sostenerse. Lo virtual desde ahora se entiende como la realidad, pero diferente. Si las funcionalidades de una tecnología no pueden ser definidas, entonces las virtualidades son las formas en que una máquina puede formar circuitos pero que no siempre se actualizan. Ideas que están en Deleuze, pero que encuentran su raíz en Gilbert Simondon. Una máquina no puede determinarse por lo que es capaz de

We are not using a metaphor, however, when we speak of machines; humans constitute a machine as soon as their nature is communicated by recurrence to the ensemble of which they form a part under specific conditions. 
hacer según los planos bajo los que fue construida. Sus virtualidades se mantienen vigentes y son parte real de la forma en que ingresan al mundo. Los computadores no fueron creados como objetos de entretenimiento, sin embargo, la posibilidad de ser usados para programar videojuegos era parte de su virtualidad y es con ella con la que los hackers formaron enlaces. «En tanto Simondon como en la cibernética, entonces, se considera lo virtual como un espacio real que queda tanto en lo real como en lo inactual ${ }^{5}$ » (Lister et al. 391, la traducción es nuetra). Lo virtual ya no puede pensarse como opuesto a lo real. Ambos son parte del mundo y se actualizan en la creación de máquinas que se conectan con sus usuarios y crean nuevos circuitos para crear nuevas máquinas tecnoorgánicas. Así se explica el carácter virtual que proponen Negri y Hardt del poder imperial en la sociedad actual, en cuanto su ejecución es completamente material al actualizar su potencialidad en los casos concretos que lo posibilitan. VR ya no es más un concepto que pueda aplicarse a una realidad simulada. Ya no se necesitan gadgets - cascos, lentes, guantes o seudocordones umbilicales- para acceder a otros mundos. El mundo es este y basta con conectar nuestro propio cuerpo a las virtualidades que ofrece la máquina, las que se actualizan de manera única en la conexión. Singularidades que se pluralizan, nuevos cuerpos-máquina que se crean y a su vez crean el mundo.

Si hay algo que ejemplifica de mejor manera esta formación de circuitos y que hoy está revolucionando la industria cultural son los videojuegos. Fueron ellos los que sentaron el camino a la computación como lugar de desarrollo de medios de entretenimiento e información, como también los que inspiraron a William Gibson a la escritura de Neuromancer. Fue el presenciar el híbrido cuerpo-máquina en los primeros arcades lo que sugirió la sensación de estar ante una nueva existencia humana. El ciberpunk nace como respuesta a la interrogante de qué pasaría con el cuerpo en el futuro una vez que las máquinas poblaran de mundos virtuales nuestra existencia, cuando la conexión no pudiera evitarse y los circuitos al estilo humanovideojuego se formaran permanentemente.

Hoy podemos ver que las consecuencias no fueron tan desastrosas como lo esperaba el ciberpunk, y, sin embargo, aún no conocemos todos los alcances que los videojuegos puedan estar teniendo en la cultura. La realidad, al menos, ya no peligra en su persistencia. Las máquinas y sus mundos virtuales forman parte indisoluble de nuestro mundo fáctico y así se ha logrado, hasta cierto punto, establecer. Las conexiones se hacen a nivel molecular: roces en el mouse, presiones en el teclado, audición, visión. No hay necesidad de puertos en nuestro cuerpo como los existentes en los personajes de Matrix. Los cuerpos actuales se mantienen intactos en su composición orgánica originaria, ya que la conexión con las máquinas se hace en pequeñísimos circuitos entre distintas partes de los diferentes cuerpos. No es un cuerpo-humano-sujeto el que se relaciona con un artefacto-computador-objeto en

In both Simondon and cybernetics, then, the virtual is considered as a real space that remains both real and inactual. 
cuanto entes completos e inalterables en su extensión. El cuerpo se divide en partes extra partes y se establece, en cada una de ellas, un microcircuito que se relaciona con otros para crear una red de tipo neuronal, como dirían los conectivistas. Los enlaces se replican infinitamente, y se conectan y desconectan para reformarse en forma simultánea y aleatoria. Algo así intuía el ciberpunk cuando cuestionaba los dualismos y presentaba mundos que contenían dentro de sí realidades contradictorias que no tenían ningún problema en coexistir. Como lo afirmaría McLuhan, la realidad se torna sincrónica en la simultaneidad de sus componentes.

Así se explica que las sesiones de juego puedan ser interrumpidas sin alterar la conexión profunda con el juego, aquella que pareciera suprimir cualquier otra conexión con el mundo. Los enlaces se arman y se replican, se repiten en su diferencia; algunos permanecen, otros se reforman, pero las virtualizaciones del juego se actualizan solo en tanto que formen un circuito. Más que interactividad, los videojuegos y los medios actuales, en general, necesitan de conexiones para existir en sus instanciaciones. Retroalimentación permanente que alimente el sistema, diría la cibernética. Ya no hay un broadcast. No hay un centro que alimente la masa. Hay multitudes, afirma Virno. De este modo, ni siquiera los noticieros pueden mantenerse hoy sin el feedback del reporteo ciudadano a través de Twitter. Existimos únicamente en la conexión comunicativa.

Los cuerpos ya no pueden ser entendidos como sacos de carne aislados que mantienen protegida a un alma. Los cuerpos son abiertos y viven en el con-tacto. Tacto simulado, dirá Baudrillard, con un interlocutor infinitamente lejano. Pero también puede afirmase tacto a secas, en cuanto el cuerpo es el que se mantiene abierto al permanente enlace maquínico con la tecnología que lo constituye y lo rodea. La téchne de los cuerpos los condena a existir únicamente cuando están conectados por el phylum maquínico que los caracteriza. El cuerpo ya no es su consistencia, su profundidad; es su límite, su roce. "No hay 'el' cuerpo, no hay 'el' tacto, no hay 'la' res extensa. Hay lo que hay: creación de mundo, téchne de los cuerpos, pesaje sin límites del sentido, corpus topográfico, geografía de las ectopías multiplicadas -y no u-topías» (Nancy, Corpus 91).

La imagen del cyborg que popularizó el ciberpunk se puede concebir como vigente cuando todavía se imagina al cuerpo como un híbrido entre naturaleza y tecnología. Pero, al igual que la realidad virtual, el concepto de cyborg ya no es como se pensó en el imaginario posapocalíptico. El cyborg ya no está constituido por la intrusión de objetos externos al cuerpo. No hay partes reemplazadas. La biotecnología y la biomecánica hoy se limitan a solucionar problemas del organismo: errores, enfermedades. Las determinaciones biopolíticas alientan la sanidad del cuerpo, la mantención de la vida, por sobre su expansión y mejoramiento. Los proyectos transhumanistas que intentan incrementar las capacidades intelectuales y físicas del ser humano no forman parte del proyecto central de las ciencias biológicas o, mejor dicho, de las políticas empresario-gubernamentales. El miedo al descontrol, a 
la sobrerreacción inmunitaria, lleva a preferir mantener la vida como la conocemos antes que a intentar crear otra diferente. El cyborg, sin embargo, se mantiene vigente como una figura (bio)política. Como una figura de resistencia.

Donna Haraway, en su Manifiesto Cyborg, propone en plena proliferación del ciberpunk una imagen del cyborg como un agente político que incorpora en su propio cuerpo las luchas que los medios de producción capitalista imponen sobre él: "Nuestros cuerpos, nosotras y nosotros mismos; los cuerpos son mapas de poder e identidad. Los cyborgs no son excepción. Un cyborg no es inocente; no nació en un jardin; no busca una identidad unitaria y asi genera dualismos antagónicos sin fin (o hasta que el mundo llegue a su fin); dan por hecho la ironía ${ }^{6}$ " (Haraway 126, la traducción es nuestra). El cuerpo cyborg es el lugar donde el cartesianismo y el dualismo platónico occidental no pueden sobrevivir. Su indeterminación racial, sexual y de especie le permite transitar por toda la gama de la identidad corpórea. Indeterminación garantizada por su carácter maquínico, su phylum que lo caracteriza como un cuerpo en constante reformación.

Esto va más allá de los usos que se le pueden dar a los nuevos medios. No se trata simplemente de crearse un avatar de mujer mientras se es biológicamente hombre. Es el constituirse en el cuerpo real como materia indeterminada, como máquina abierta a la actualización de sus virtualidades universales. Los cuerpos son construidos técnicamente y el biopoder tiende a normalizar y controlar esas construcciones hacia estándares que permitan el mejor flujo de trabajo y la mantención de la vida. Sin embargo, esa misma característica, que permite el control imperial, permite también la libertad de asumir los medios técnicos de producción de cuerpo y construirlos fuera de la norma como medio de resistencia. Más que mal, con Foucault existe la noción de que todo poder está compuesto necesariamente de fuerzas opuestas. Para que exista poder debe haber resistencia. La resistencia contemporánea está en la construcción maquínica de los cibercuerpos / cyborgs. Después de todo, el ciberpunk no es puro pesimismo. El lugar que le corresponde a los seguidores del ciberpunk será el de la fuerza de disidencia, de ahí el concepto de «punk». Los que se identifican con este movimiento serán los rechazados de la sociedad, las minorías que ven en la tecnología un medio mediante el cual revelarse. No tratan necesariamente de evitar este tipo de futuro, pues tal vez puedan encontrar una ventaja. Y, así, el tema de la fusión entre hombre y tecnología será recurrente: los cyborgs serán el posible futuro de la humanidad. Incluso, para algunos, aquel será el mejor futuro que pueda tener el ser humano, un mañana en el que las limitaciones del hombre serán superadas gracias a la manipulación técnica. El límite entre lo artificial y lo natural será cada vez más difuso y, como consecuencia, lo será también la división entre virtualidad y realidad, el mundo creado por las máquinas y el que habitan los humanos. El sobreviviente será quien logre moverse libre e indistintamente por estos mundos o,

6 Our bodies, ourselves; bodies are maps of power and identity. Cyborgs are no exception. A cyborg is not innocent; it was not born in a garden; it does not seek unitary identity and so generate antagonistic dualisms without end (or until the world ends); it takes irony for granted. 
mejor aún, quien logre juntarlos: el gamer. Será así que el papel protagónico en las historias ciberpunk se le asignará a personas o grupos marginados de la sociedad controlada por las máquinas o las figuras de poder que las utilizan: corporaciones. El hacker será el antihéroe por antonomasia al tener la capacidad de burlar las barreras impuestas por la tecnología, al ocupar esas mismas herramientas y volverlas contra los grupos de poder.

Sin embargo, Haraway cuestiona el mundo de los videojuegos por fomentar patrones masculinos y heteronormativos de comportamiento: competencias e individuaciones de índole capitalista. Sin embargo, es en los mismos videojuegos donde de mejor manera puede entenderse que el cuerpo se construye técnicamente y crea otros escenarios a través de la técnica. Lidia con ella y a la vez se asume como tal. Helen W. Kennedy, por ejemplo, estudia a las mujeres que juegan un videojuego sumamente masculino para los patrones normativos, Quake, que trata básicamente de un FPS (First Person Shoot-em-up), en el que se deben asesinar extraterrestres bajo la perspectiva de una cámara en primera persona con distintas armas de fuego. Es un juego de guerra. El mercado caracteriza al público objetivo de este tipo de juegos como necesariamente masculino y, por lo tanto, lo distribuye y visibiliza por los canales que lleguen a ese tipo de público. Surge entonces el problema de que una mujer, comúnmente, no adquiere las competencias -bastante especializadas- para dominar un FPS. No se le permite el acceso a los juegos, pues en sus hogares y lugares de estudio la conexión está dominada por hombres. Las mujeres que logran acceder, sin embargo, siguen las mismas reglas y pueden entrar a un nivel competitivo equivalente al de sus pares masculinos. En ese momento entienden su cuerpo como una construcción que pueden modificar en el gameplay, en el instante cuando adquieren las competencias predeterminadas por la bionormatividad para el género masculino. Se genera, entonces, una resistencia en la posibilidad técnica de asumir el cuerpo en formas disidentes a las que la máquina imperial produce. Las virtualidades de las nuevas tecnologías permiten construir circuitos que no pueden ser previstos por la administración dominante. El éxodo que proponen Negri y Hardt finalmente se da en la apropiación de las tecnologías como lugar de autocreación y actualización de las virtualidades que aquellas posibilitan. Los cuerpos existen en la creación, una idea ya mencionada por Nietzsche, que hoy adquiere las posibilidades técnicas para realizarse.

Para comprender la sociedad contemporánea no es factible analizar la relación entre cuerpo y máquina o entre ser humano y tecnología. «La pregunta que nos hemos de hacer no es sobre el humano y la tecnología, pero sobre el humano como tecnología $»^{7}$ (Wise 196). No la relación, sino lo que los caracteriza a ambos como entes que no pueden comprenderse ya el uno sin el otro. Lo postorgánico o posthumano no es más que la imposibilidad de un humanismo que pueda definirse en contra de la tecnología. En un paradigma biopolítico no es posible entender la vida fuera de

The question we should ask shouldn't be of the human and technology, but of the human as technology. 
la técnica que crea los cuerpos-máquina por los que fluye. Así, cualquier alarmismo que subraye la ingeniería genética o la biomecánica, como el trágico final de lo que constituía ser humano, está faltando gravemente a la oportunidad de comprender a la sociedad actual en la multifocalidad de circuitos que se forman y crean resistencias.

Antes de reaccionar ante la posibilidad de un mundo genéticamente manipulado en el que se discrimina a ciertas personas por no ser perfectas biológicamente, como en el filme Gattaca de Andrew Niccol, es preciso considerar las reales herramientas que el biopoder entrega a los múltiples cuerpos que crean cuando no tienen otra forma de existir que creando a través de su conexión maquínica. La guerra por los derechos de autor desde la caída de Megaupload parece ser un escenario en el que cada grupo de poder pone a prueba su dominio sobre la arquitectura de Internet. Piratebay propone la puesta aérea de satélites que permitan la construcción de una red alternativa fuera del dominio de las políticas territoriales. La tecnología siempre va a permitir un escape, un hackeo. Lo importante, entonces, es no limitar los nuevos medios a su «virtualidad» como si ella fuera opuesta a lo que pasa IRL (in real life, según la jerga informática). La realidad también se puede hackear si se comprende que las nuevas tecnologías son, en efecto, agentes materiales que modifican y son modificados por los cuerpos orgánicos con los que cohabitan y se conectan. La ecotecnia de los cuerpos no es la ingeniería genética, ahí no hay cuerpos. Los cuerpos viven. Los cuerpos sangran todavía. Los cuerpos tocan y se tocan. Los cuerpos desean y en ese deseo configuran, en cada instancia, el mundo. Se repiten y se multiplican. Crean otros cuerpos. Otros se destruyen. ¿Con qué fundamento se puede afirmar la superación de lo orgánico, lo obsoleto de los cuerpos?

\section{LOS ESTUDIOS DEL CUERPOY LA CULTURA}

To understand contemporary net based media one must spend time online, not reading books.

Lister et al.

El cuerpo-máquina en su capacidad de creación irrumpe los cánones disciplinarios que se aíslan en un campo de estudios propio que no logra apuntar hacia las nuevas formas en que nuestra cultura se está desarrollando.

Bruno Latour ha notado, en su análisis antropológico de las ciencias, que revistas y publicaciones transitan indistintamente entre ámbitos del conocimiento que antes parecían pertenecer a mundos diferentes. Esta «proliferación de híbridos» pasa, sin embargo, desapercibida ante la parcelación disciplinar. A pesar de que un artículo sobre el calentamiento global trate de dinámicas de población humana y animal bajo los mismos términos, comente sobre la emanación de gases de fábricas y automóviles, y termine relatando la convención de la ONU anual al respecto, se sigue considerando al artículo como parte de una sección particular, en este caso, «medioambiente». «Nuestra vida intelectual está fuera de sí. La epistemología, las ciencias sociales, 
las ciencias de los textos -todas tienen su privilegiado punto de ventaja siempre y cuando se mantengan separadas. Si las criaturas que estamos persiguiendo cruzan los tres espacios, ya no somos comprensibles ${ }^{8}$ " (Latour, The Proliferation of Hybrids 108, la traducción es nuestra). Sin tomar las medidas apropiadas para que esta falta de entendimiento se supere, jamás se podrá comprender un escenario tan complejo como el del cuerpo en la actualidad.

El cuerpo se escapa, se vuelve a indefinir en cada paso. Por algo Nancy solo presenta indicios, no hechos o rasgos. El indicio, el índice, enumeran indefinidamente una proliferación de los cuerpos y su constitución técnica. Los cuerpos no son solo textos, los cuerpos no son solo ciencia, los cuerpos ni siquiera son solo cuerpos.

Y cuando nuestra política, nuestros medios de producción, nuestros tratados internacionales y nuestros proyectos en medioambiente, todos pasan irrevocablemente por la medida del cuerpo, es insostenible mantenerse en su parcelación, su encarcelamiento, su desmembramiento en disciplinas dispares. Para las humanidades, y la estética en particular, esto resulta un problema grave. Cuando la cultura se entiende solo a partir de la lectura del discurso, la representación, el lenguaje, el texto, la retórica; cuando se ignoran las materialidades del cuerpo y aquellas con las que el cuerpo se relaciona, entonces no hay posibilidad de acercarse a la cultura.

«Será que la sociedad misma ha de ser repensada de pies a cabeza una vez que le hayamos añadido los hechos y los artefactos que componen gran parte de nuestros lazos sociales» ${ }^{9}$ (Latour, Where Are the Missing Masses? 151, la traducción es nuestra).

Las masas perdidas de las que habla Latour están hoy frente a nuestros ojos ocupando gran parte de lo que constituye la cultura. Y no basta con acercarse a estos artefactos con la ingenuidad de las humanidades. No basta mirar un computador y observar cómo el usuario se comporta frente a él. Es necesario entender el artefacto en sí, comprender su funcionamiento, su arquitectura de hardware y software. No se trata tampoco de que todos los humanistas se hagan ingenieros, ni que todos los ingenieros se enfoquen hacia los estudios culturales, pero los cambios que está experimentando la sociedad deben ser atendidos interdisciplinariamente. Es necesario establecer lenguajes comunes, propuestas reales de trabajo conjunto.

Y entre las masas perdidas no solo están las máquinas. También están los cuerpos. O como ya se ha establecido, están los cuerpos-máquinas. Los cyborgs contemporáneos que por las ciencias biológicas son tomados como objetos de ingeniería y por las humanidades son articulados como texto, cuando no son solamente lo uno o lo otro. Si el cuerpo es un híbrido, entonces aparecerá en el cruce de distintas aproximaciones simultáneas. Su genética, su lengua, su performance. Todas ellas son parte

8 Our intellectual life is out of kilter. Epistemology, the social sciences, the sciences of texts -all have their privileged vantage point, provided that they remain separate. If the creatures we are pursuing cross all three spaces, we are no longer understood.

9 It is that society itself is to be rethought from top to bottom once we add to it the facts and the artifacts that make up large sections of our social ties. 
del cuerpo, es decir, todas ellas son cuerpo, sin que una pueda ser separada de la otra. En la separación ya no hay cuerpo. Hay cadáveres.

La Estética, por su parte, aquella disciplina encargada de la aisthesis, de la percepción del mundo en el cuerpo, es la que debería estar a la vanguardia de esta nueva forma de comprender la cultura; sin embargo, se limita a los usos del cuerpo, a sus distribuciones, a sus movimientos, a sus gritos, saltos y actuaciones. Estudios de performance todos. Pero los cuerpos no son eso, no es eso lo que los constituye como cultura. Reducir al cuerpo al uso que le otorga un sujeto sería intentar estudiar las redes sociales por las estadísticas demográficas de acceso, por los «me gusta» más numerosos, por las páginas más visitadas, por las personas con las que interactúan. Pero hay un cuerpo haciendo funcionar la red, creando la red en su funcionar. Hay una interfaz, hay un software, una página web que permite y coarta -cibernéticamente- un comportamiento. Hay un feedback, hay un circuito entre la interfaz de la red social y la percepción del mundo. Está la dimensión física que permite la disposición corpórea del enlace cuerpo-máquina. Estas deberían ser las preocupaciones de la Estética hoy: observar un cuerpo que percibe el mundo de forma diferente, un cuerpo que se percibe diferente.

Mas estas proposiciones no son arbitrarias. El propósito de este ensayo radica en demostrar que ciertas temáticas son transversales y centrales a las ciencias sociales y las humanidades. Que la Estética deba preocuparse del cuerpo y las máquinas que lo rodean en la contemporaneidad no es una sentencia únicamente aplicable a aquellos que se dedican a los estudios de nuevos medios y culturas digitales. Cuando la percepción del mundo ha cambiado, entonces no hay área de las artes y la cultura que no esté afectada por ello.

La perspectiva biopolítica sirve, en este sentido, para constatar la importancia del cuerpo en cuanto tecnología en las dinámicas actuales de la sociedad en su conjunto. Es a partir del estudio de los flujos del biopoder que se encuentra un escenario en que el cuerpo puede ser considerado el centro y el inicio de los circuitos comunicativos que sustentan la legitimidad del régimen de la vida. Un régimen que seguirá activo mientras la política esté atravesada por el bíos. Políticas que, siempre es bueno dejar en claro, no corresponden exclusivamente a dictámenes administrativos guiados por los grupos que concentran el poder económico y político. La resistencia también está atravesada por el bíos y es en esa vida, en ese bíos constituido simultáneamente por la téchne y la zoé, donde la lucha de poder se concentra mientras se habita el mundo como cuerpo-máquina. Tecnocuerpo siendo construido y construyendo sin que exista la posibilidad de determinar quién es la mente que planea tales movimientos de creación. La mente también es cuerpo.

Pero no se trata tampoco de volver a los viejos utopismos que proponen las nuevas tecnologías como herramientas que cambiarán el mundo de tal forma que la democracia, el trabajo y las libertades evolucionarán a límites insospechados. El análisis biopolítico de este ensayo permite considerar también los abusos y los alcances 
que las mismas tecnologías tienen para sustentar la sociedad posfordista en la que estamos insertos. «No es necesaria la ciencia ficción para concebir un mecanismo de control que señale a cada instante la posición de un elemento en un lugar abierto, animal en una reserva, hombre en una empresa» (Deleuze 3). La idea de que entregar computadores en sectores de pobreza automáticamente mejorará el nivel educativo y las oportunidades de superación social responde a un determinismo tecnológico que, desde cualquier punto de vista, debe rechazarse. Lo mismo ocurre con pretender que el acceso libre a Internet en China entregaría necesariamente una democracia -neoliberal- al país. Las tecnologías biopolíticas permiten irrestrictivamente a los grupos que concentran el poder económico y político administrar las máquinas y los cuerpos de acuerdo a sus intereses, ya que el desarrollo técnico y la distribución de la tecnología son promovidos por los mismos organismos interesados. No obstante, es la misma arquitectura de las tecnologías que sustentan a la sociedad de control lo que permite, como dirán Negri y Hardt, una salida:

Primero, cada lucha, aunque firmemente asentada en condiciones locales, salta de inmediato al nivel global y ataca a la constitución imperial en su generalidad. Segundo, todas las luchas destruyen la distinción tradicional entre luchas políticas y económicas. Estas luchas son, a un mismo tiempo, económicas, políticas y culturales -y, por lo tanto, son luchas biopolíticas, luchas sobre la forma de vida. Son luchas constituyentes, creando nuevos espacios públicos y nuevas formas de comunidad (49).

Lo importante es aceptar que las tecnologías no son intrínsecamente perjudiciales o beneficiosas. Las virtualidades de una máquina permanecen siempre indeterminadas hasta que su contacto con la sociedad las va actualizando. Por lo tanto, ni los grupos que concentran el poder ni las luchas de resistencia pueden asumir que una tecnología por sí sola conducirá a tales o cuales resultados. No son Facebook ni Twitter los que han generado las revueltas alrededor del mundo en los últimos años, sino la disposición de los cuerpos a utilizar las tecnologías para crear una diferencia en el plano material en el que están insertos cuando se conectan a aquellas redes sociales. De hecho, podría incluso decirse que la aparente libertad de Twitter permite crear la ilusión de que las libertades sociales son cultivadas, cuando en realidad los Trending Topic son cuidadosamente monitoreados para ejercer control sobre la visibilidad de los temas discutidos. Así, la efervescencia de una revuelta social generada por Internet es rápidamente aplacada e ilusoriamente satisfecha en cuanto la visibilidad puede ser modificada.

En consecuencia, estudiar las tecnologías biopolíticas es de vital importancia para considerar las distintas estrategias que los diversos grupos de poder pueden ejercer en la forma en que actualizan las virtualidades de las máquinas-cuerpos que configuran. Considerar al humano un cyborg que existe en el momento en que logra formar un circuito con una máquina que lo conecte a la red informativa, permite tomar conciencia de que ese mismo circuito puede ser manipulado. Una vez se ha establecido la naturaleza de la conexión, la resistencia puede crearse en la aplica- 
ción de contrafuegos a los posibles ataques que la máquina imperial lanza contra los cuerpos. El ciberpunk, en este sentido, pareciera no estar tan obsoleto. Una vez superado el miedo a las máquinas, una vez asumida su condición de compañeros de phylum, es posible abrir infinitas opciones a la reconfiguración de la vida. Tal como el protagonista de una historia ciberpunk, corresponde al sujeto contemporáneo -y a las disciplinas que los estudian- armarse del conocimiento físico y teórico de las técnicas que configuran al mundo y a su propio cuerpo. Algo que siempre ha sido cierto, pero que en la sociedad actual prolifera en la capacidad tecnológica de una conexión permanente y una sincronía de microcircuitos. Una lucha que no es simplemente externa, sino que se atrinchera en los límites del cuerpo.

Si todo esto implica colocar un "post» antes del vocablo "humano», pues bienvenida sea la ola que borre el nombre del hombre una vez escrito en la arena.

\section{REFERENCIAS}

Baudrillard, Jean. "The Vanishing Point of Communication.» The New Media and Technocultures Reader. Ed. Seth Giddings \& Martin Lister. London: Taylor and Francis, 2009. Medio impreso.

Bell, David. Cyberculture Theorists: Manuel Castells and Donna Haraway. London: Taylor \& Francis, 2007. Medio impreso.

Berman, Marshall. Todo lo sólido se desvanece en el aire: la experiencia de la modernidad. Madrid: Siglo XxI, 1989. Medio impreso.

Cameron, James, dir. The Terminator. 1984. Medio fílmico.

Cronenberg, David, dir. eXistenZ. 1999. Medio fílmico.

Deleuze, Gilles. «Postdata sobre las sociedades de control». El lenguaje literario. Ed. Christian Ferrer. Vol. 2. Montevideo: Nordan, 1991. Medio impreso.

Esposito, Roberto. Bíos. Biopolítica y filosofía. Buenos Aires: Amorrortu, 2006. Medio impreso.

Foucault, Michel. El orden del discurso. Buenos Aires: Tusquets Editores, 1992. Medio impreso.

---. Historia de la sexualidad: La voluntad de saber. I. Madrid: Siglo xxi, 2005. Medio impreso.

---. Las palabras y las cosas: una arquelogía de las ciencias humanas. Madrid: Siglo XXI, 1989. Medio impreso.

---. Vigilar y castigar. Madrid: Siglo xxi, 2000. Medio impreso.

Guattari, Félix. «Balance Program for Desiring-machines.» The New Media and Technocultures Reader. Ed. Seth Giddings \& Martin Lister. London: Taylor and Francis, 2009. Medio impreso.

Haraway, Donna. «Selected Material from 'A Cyborg Manifesto'». The New Media and Technocultures Reader. Ed. Seth Giddings \& Martin Lister. London: Taylor and Francis, 2009. Medio impreso. 
Kennedy, Helen W. «Female Quake Players and the Politics of Identity». The New Media and Technocultures Reader. Ed. Seth Giddings \& Martin Lister. London: Taylor and Francis, 2009. Medio impreso.

Latour, Bruno. "The Proliferation of Hybrids.» The New Media and Technocultures Reader. Ed. Seth Giddings \& Martin Lister. London: Taylor and Francis, 2009. Medio impreso.

---. «Where Are the Missing Masses? The Sociology of a Few Mundane Artifacts.» The New Media and Technocultures Reader. Ed. Seth Giddings \& Martin Lister. London: Taylor and Francis, 2009. Medio impreso.

Lazzarato, Maurizio. «Del biopoder a la biopolítica». otrascosas. Fecha de ingreso: 27 de marzo de 2010. <http://bit.ly/6nOexz>. Sitio web.

---. «Immaterial Labour.» Theory Out of Bounds. Radical Thought in Italy, a Potential Politics. Minneapolis: Minnesota University Press, 1996. Medio impreso.

Lisberger, Steven, dir. Tron. 1982. Medio fílmico.

Lister, Martin et al. New Media: a Critical Introduction. London: Taylor \& Francis, 2009. Medio impreso.

Marx, Karl. «The Labour Process and Alienation in Machinery and Science.» The New Media and Technocultures Reader. Ed. Seth Giddings \& Martin Lister. London: Taylor and Francis, 2009. Medio impreso.

McLuhan, Marshall. «Selected Material from 'Understanding Media: The Extensions of Man'». The New Media and Technocultures Reader. Ed. Seth Giddings \& Martin Lister. London: Taylor and Francis, 2009. Medio impreso.

Morales, Hugo. «Ex ejecutivo de Google asegura que Larry Page ha arruinado a la empresa - FayerWayer». FayerWayer. Fecha de ingreso: 14 de marzo de 2012. $<$ http://bit.ly/zW6MWW>. Sitio web.

Nancy, Jean-Luc. 58 indicios sobre el cuerpo. Extensión del alma. Buenos Aires: La Cebra, 2007. Medio impreso.

---. Corpus. Madrid: Arena Libros, 2003. Medio impreso.

Negri, Toni y Michael Hardt. «Imperio». Chile Vive, 2000. Fecha de ingreso: 12 de diciembre de 2012. Sitio web.

Gattaca, Niccol, Andrew, dir. 1997. Medio fílmico.

Proyas, Alex, dir. I, Robot. 2004. Medio fílmico.

Scott, Ridley, dir. Blade Runner. 1982. Medio fílmico.

SFAM. «What is Cyberpunk?». Cyberpunkreview. <http://bit.ly/5zNjO0>. Sitio web.

Sibilia, Paula. El hombre postorgánico: cuerpo, subjetividad y tecnologías digitales. Buenos Aires: Fondo de Cultura Económica, 2005. Medio impreso.

Temple, Krystal. «What Happens in an Internet Minute?». Inside Scoop. Fecha de ingreso: poner la fecha. <http://intel.ly/A02bcJ>. Sitio web.

Tomas, David. «Feedback and Cybernetics: Reimaging the Body in the Age of The Cyborg." The New Media and Technocultures Reader. Ed. Seth Giddings \& Martin Lister. London: Taylor and Francis, 2009. Medio impreso. 
Ulloa, Gabriela. «Estudio muestra que la mayoría del tráfico de Internet no es humano». BioBioChile. Fecha de ingreso: 19 de marzo se 2011. <http://bit.ly/FSeyXS> Sitio web.

Virno, Paolo. Gramática de la multitud. Madrid: Traficantes de Sueños, 2003. Medio impreso.

Wachowki, Andy y Lana Wachowki, dir. The Matrix. 1999. Medio fílmico.

Williams, Raymond. "The Technology and the Society.» The New Media and Technocultures Reader. Ed. Seth Giddings \& Martin Lister. London: Taylor and Francis, 2009. Medio impreso.

Wise, J. Macgregor. «Intelligent Agency.» The New Media and Technocultures Reader. Ed. Seth Giddings \& Martin Lister. London: Taylor and Francis, 2009. Medio impreso. 